\title{
A RAKE Structured SINR Maximizing Mobile Receiver for the WCDMA Downlink
}

\author{
Massimiliano Lenardi Dirk T.M. Slock \\ Institut Eurécom*, 2229 route des Crêtes, B.P. 193, \\ 06904 Sophia Antipolis Cedex, FRANCE \\ Tel: +33 49300 2910/2606; Fax: +33 493002627 \\ e-mail: $\{$ lenardi, slock\}@eurecom.fr
}

\begin{abstract}
We propose a RAKE structured receiver with the pulseshape MF replaced with another FIR filter, and the sparse propagation channel MF by another sparse filter. We compare different choices for the design of the FIR filter and the sparse filter and their adaptation. We model the channel as an autoregressive first-order process over slot periods, with bandwidth commensurate with the Doppler spread, and we refine the brute pilot-based slot-wise estimate using adaptive causal Wiener filtering across slots. We then apply recursive path extraction in every slot. We also introduce polynomial expansion extensions, one at chip rate to improve the MMSE equalization performance of the structured cip rate equalizer, and one at symbol rate to bring the structured receiver performance closer to that of the global time-varying LMMSE receiver.
\end{abstract}

\section{1: Introduction}

This paper focuses on the DS-CDMA FDD-mode downlink of the 3GPP UMTS proposal. In [6] we proposed a generalized linear receiver, the max-SINR receiver, which encompasses the RAKE and the equalizer-plus-correlator receivers [2] as special cases. The structure is the same of the RAKE receiver, but the channel and pulse shape matched filters are replaced by an equalizer filter that is designed to maximize the SINR at the output of the receiver. However, the complexity of running the MMSE equalizer at chip rate is much higher than performing pulse shape matched filtering at chip rate followed by sparse propagation channel matched filtering (recombining) at symbol rate. Therefore, in [7] we studied different lower-complexity implementations of the equalizer, including a cascade of a pulse

\footnotetext{
*Eurécom's research is partially supported by its industrial partners: Ascom, Swisscom, Thomson-CSF, IBM France, CEGETEL, Motorola, France Télécom, Hitachi Europe and Texas Instruments.
}

shape matched filter and a sparse filter, whose coefficients were optimized to maximize the output SINR. In [4] the case of a mobile terminal equipped with multiple sensors, has been studied. The equalizer simply becomes a spatiotemporal MMSE equaliser. In this paper we propose the application of polynomial expansion, see [10], to the reduced complexity versions of this max-SINR receiver and their adaptation. The Linearly Constrained Minimum Variance (LCMV) adaptation can be done in a semi-blind fashion at symbol rate using all symbol periods, while requiring the same information (channel estimate, which can be obtained using pilot information) as the RAKE receiver. The mobile channel is modeled as a first-order AR process and channel estimation is performed by Recursive Early-late, see [8], on a pilot-based channel estimate, refined by adaptive optimal Wiener filtering across slots.

\section{2: Multiuser Downlink Signal Model}

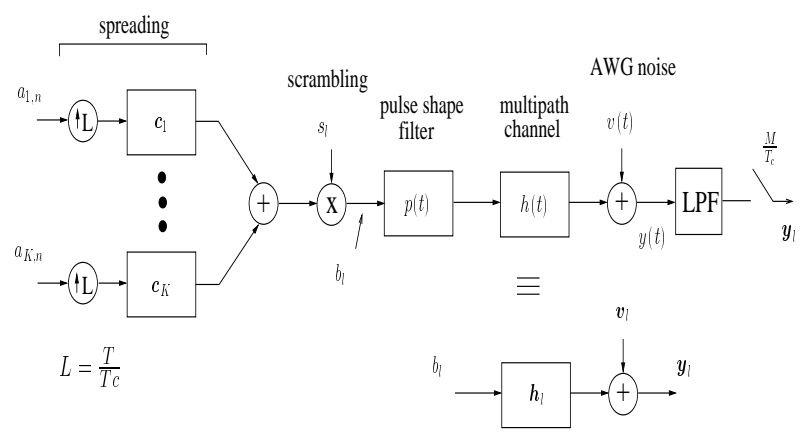

Figure 1. Downlink signal model BS-MS

Fig. 1 shows the downlink signal model in baseband. Stacking the $M$ samples per chip period in vectors, we get for the sampled received signal at MS antenna $j$ during chip pe$\operatorname{riod} l$

$$
\boldsymbol{y}_{l}^{j}=\sum_{k=1}^{K} \sum_{i=0}^{N-1} \boldsymbol{h}_{i}^{j} b_{k, l-i}+\boldsymbol{v}_{l}^{j}, \quad j=1, \ldots, J .
$$


Here $\boldsymbol{y}_{l}^{j}, \boldsymbol{h}_{l}^{j}$ and $\boldsymbol{v}_{l}^{j}$ represent the vectors at the chip rate of samples at the sampling rate. $\boldsymbol{h}_{l}^{j}$ denotes the overall channel, including pulse shape, propagation channel and receiver filter for the MS antenna $j(j=1 \cdots J)$. The overall channel is assumed to have a delay spread of $N$ chips due to contributions from $P$ paths. The multipath description of the channel for oversampling phase $m$ at antenna $j$ and during chip period $l$ is

$$
h_{m, l}^{j}=\sum_{p=1}^{P} \alpha_{p}^{j} p\left(l T_{c}+\frac{(m-1) T_{c}}{M}-\tau_{p}\right)
$$

For antenna $j,\left\{\alpha_{p}^{j}\right\}$ is the complex amplitude of path $p$ with corresponding delay $\left\{\tau_{p}\right\}$ (the delays for a given path are equal for all $J$ MS antennas). If we model the scrambling sequence and the symbol sequences as independent i.i.d. sequences, then the chip sequence $b_{l}$ is a sum of $K$ independent white noises (chip rate i.i.d. sequences, hence stationary). The intracell contribution to $\boldsymbol{y}_{l}^{j}$ then is a stationary (vector) process (the continuous-time counterpart is cyclostationary with chip period).

\section{3: Max-SINR Receiver Structure}

As shown in Fig. 2, the receiver is constrained to be a chip rate filter $f$ followed by a descrambler and a correlator with the spreading code of the user of interest, which is here assumed to be user 1. So the receiver has the same structure as a RAKE receiver, except that the channel matched filter gets replaced by a general filter $f$. If a sparse (path-wise) representation is used for the channel, then the channel matched filter leads to a RAKE structure with one finger per path. In Fig. 2, the operation "S/P" denotes a serial to parallel conversion which stacks the $L$ most recent inputs into a vector. The correlator can also be viewed as a matched filter, matched to the spreading code filter, but here it is simply depicted as an inner product on a downsampled vectorized signal. The analysis done in [6] shows that, due to the or-

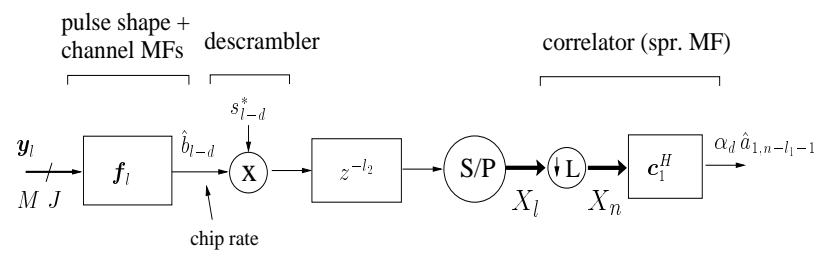

Figure 2. The downlink receiver structure

thogonality of the spreading codes and to the i.i.d. character of the scrambler, the SINR, $\Gamma$, at the receiver output is

$$
\Gamma=\frac{\sigma_{1}^{2}|\boldsymbol{f} \boldsymbol{h}|^{2}}{\boldsymbol{f} R_{Y Y} \boldsymbol{f}^{H}-\sigma_{t o t}^{2}|\boldsymbol{f} \boldsymbol{h}|^{2}}
$$

where $\sigma_{k}^{2}=\mathrm{E}\left|a_{k, n}\right|^{2}, \sigma_{t o t}^{2}=\frac{1}{L} \sum_{k=1}^{K} \sigma_{k}^{2}$ and

$R_{Y Y}=R_{V V}+\sigma_{\text {tot }}^{2} \mathcal{T}\left(\boldsymbol{h}^{\prime}\right) \mathcal{T}^{H}\left(\boldsymbol{h}^{\prime}\right)$ where $\mathcal{T}\left(\boldsymbol{h}^{\prime}\right)$ denotes a block Toeplitz convolution matrix and $\boldsymbol{h}^{\prime}$ denotes $\boldsymbol{h}=$ $\left[\boldsymbol{h}_{0}^{T} \cdots \boldsymbol{h}_{N-1}^{T}\right]^{T}$ but with its chip rate coefficients in reversed order. The choice for the filter $f$ that leads to maximum receiver output SINR is unique up to a scale factor and can be found as the solution to the following problem

$$
\begin{aligned}
\boldsymbol{f}_{M A X} & =\arg \max _{\boldsymbol{f} \boldsymbol{f} \boldsymbol{h}=1} \Gamma=\arg \min _{\boldsymbol{f}: \boldsymbol{f} \boldsymbol{h}=1} \boldsymbol{f} R_{Y Y} \boldsymbol{f}^{H} \\
\Rightarrow \boldsymbol{f}_{M A X} & =\left(\boldsymbol{h}^{H} R_{Y Y}^{-1} \boldsymbol{h}\right)^{-1} \boldsymbol{h}^{H} R_{Y Y}^{-1}
\end{aligned}
$$

The SINR becomes

$$
\Gamma_{M A X}=\frac{\sigma_{1}^{2}}{\left(\boldsymbol{h}^{H} R_{Y Y}^{-1} \boldsymbol{h}\right)^{-1}-\sigma_{t o t}^{2}}
$$

As pointed out in [6], this receiver corresponds to the cascade of an unbiased MMSE receiver for the desired user's chip sequence, followed by a descrambler and a correlator. In the noiseless case, the MMSE receiver $\boldsymbol{f}_{M A X}$ becomes a ZF equalizer. In fact, the max-SINR receiver is related to the linear MMSE receiver which is

$$
\widehat{a}_{1}=R_{a_{1} Y} R_{Y Y}^{-1} Y=\sigma_{a}^{2} \boldsymbol{c}_{1}^{H} S^{H} \mathcal{T}^{H}(\boldsymbol{h}) R_{Y Y}^{-1} Y
$$

where we omitted time indices and we assume that the FIR LMMSE is based on a stretch of signal $Y . S$ is a diagonal matrix containing the scrambling sequence over a certain symbol period, $R_{a_{1} Y}=\mathrm{E}_{a, v} a_{1} Y^{H}, R_{Y Y}=\mathrm{E}_{a, v} Y Y^{H}$ where $\mathrm{E}_{a, v}$ denotes expectation over symbols and noise. Due to the scrambling sequence, $R_{Y Y}$ is time-varying and the whole LMMSE is time-varying. The max-SINR is obtained when $R_{Y Y}$ is computed using $\mathrm{E}_{a, v, s}$, so by averaging over the scrambler also, considered as an i.i.d. sequence. In that case $\sigma_{a}^{2} \mathcal{T}^{H}(\boldsymbol{h}) R_{Y Y}^{-1}$ is of the form $\mathcal{T}(\boldsymbol{f})$ where $f$ is an MMSE (chip rate) equalizer. So the LMMSE receiver becomes a MMSE equalizer-descrambler-correlator cascade.

\section{4: Structured Equalizer Receivers}

In [4] we presented 5 structured max-SINR multi-sensor receivers: the pulse-shape matched filter (GRAKE), the path-wise equalizer (PWEQRAKE) and its averaged and per-antenna reduced complexity versions (AWEQRAKE and PAEQRAKE) and finally the joint iterative (alternating) equalizer (JIEQRAKE). Their structure arises when we impose to the equalizer to be a cascade of a short spatiotemporal FIR filter followed by a sparse spatio temporal filter, see Fig. 3, and we choose and/or optimize differently the taps of the short FIR filter and/or the coefficients of the sparse filter, optimizing to maximize the output SINR.

Therefore, the general structure for these receivers is depicted in Fig. 4.

For the simulations here, we keep the GRAKE, for which the pulse-shape equalizer $F$ is simply the Root-RaisedCosine (RRC) matched filter (as in the RAKE) and the 


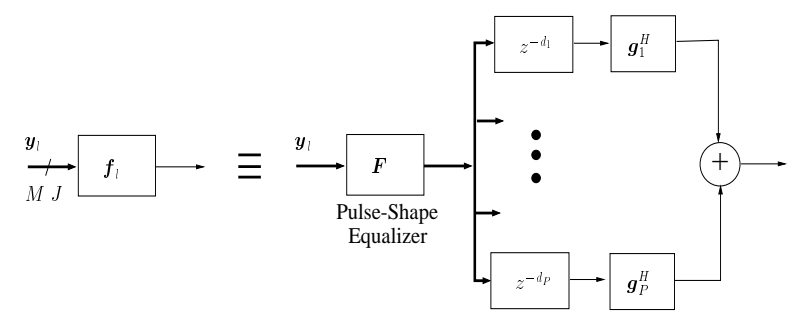

Figure 3. The structured equalizer

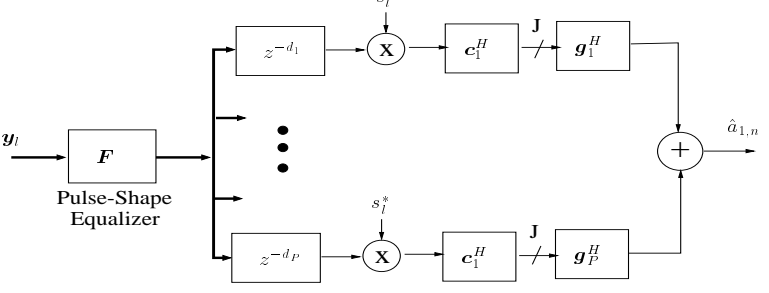

Figure 4. The path-wise equalizer RAKE structure

sparse filter coefficients are optimized for max SINR, and we keep the JIEQRAKE, which optimizes, alternatingly, both filters, but $F$ has in this case just different temporal filters for each antenna (not full spatiotemporal). We will compare these two equalizers with the unstructured FIR max-SINR equalizer (4) and with the RAKE.

\section{5: Polynomial Expansion Extensions}

The work done in [5] (see also [10] for background) allows us to apply the chip-rate Polynomial Expansion (PE) theory to the path-wise receiver structures of previous section. We can write the received chip-rate signal $\boldsymbol{y}_{l}=$ $\boldsymbol{H}(z) b_{l}+\boldsymbol{v}_{l}$, where $\boldsymbol{H}(z)$ is the channel transfer function at chip rate. The equalizer output $\boldsymbol{x}_{l}=\boldsymbol{F}(z) \boldsymbol{x}_{l}$ can be rewritten as $\boldsymbol{x}_{l}=\boldsymbol{D}(z) b_{l}+\boldsymbol{F}(z) \boldsymbol{v}_{l}$, where $\boldsymbol{D}(z)=\boldsymbol{F}(z) \boldsymbol{H}(z)$, and an estimate of the user of interest (user 1) symbol can be obtained by $\hat{a}_{1}(n)=\boldsymbol{c}_{1}^{H} \boldsymbol{S}_{n}^{H} \boldsymbol{D}^{-1}(z) \boldsymbol{X}_{n}$, being $\boldsymbol{X}_{n}$ a concatenation of $\boldsymbol{x}_{l}$ over a symbol period. Computation of the exact inverse of $\boldsymbol{D}(z)$ would lead to high complexity, so we can approximate it by polynomial expansion, assuming that the main tap ( $\left.\alpha_{d}=\alpha_{0}=1\right)$ of $\boldsymbol{D}(z)$ is dominant in magnitude over all the other taps (close to zero forcing), $D^{-1}(z)=\sum_{i=0}^{\infty}(\boldsymbol{I}-\boldsymbol{D}(z))^{i}$. As a result, an estimate of the user of interest symbols, at first order polynomial expansion, can be rewritten as $\hat{a}_{1}(n)=\boldsymbol{c}_{1}^{H} \boldsymbol{S}_{n}^{H} \overline{\boldsymbol{F}}(z) \boldsymbol{Y}_{n}$ with $\overline{\boldsymbol{F}}(z)=\boldsymbol{F}(z)(2 \boldsymbol{I}-\boldsymbol{H}(z) \boldsymbol{F}(z))$. The corresponding implementation is depicted in Fig. 5. Both filters $f$ 's can be structured and optimized jointly for max SINR. Matrix $\Pi=I$ for chip-rate $\mathrm{PE}$, while for symbol-rate $\mathrm{PE}$ $\Pi=\boldsymbol{S}_{l}^{H} \boldsymbol{P} \boldsymbol{S}_{l}$, where $S$ is the scrambler and $\boldsymbol{P}$ projects on the used codes subspace.

\section{6: Channel Estimation}

In [9] we consider the estimation of mobile channels that are modeled as autoregressive processes with a band-

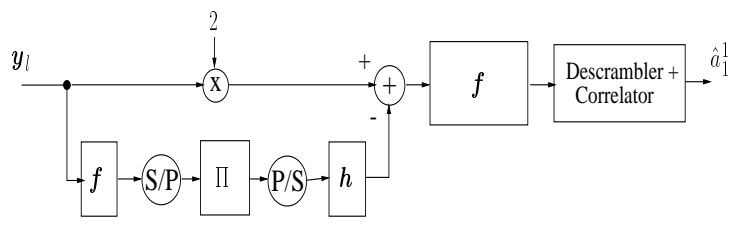

Figure 5. Chip Rate Polynomial Expansion structure

width commensurate with the Doppler spread. Pilot based estimation leads to brute FIR channel estimates $\hat{\boldsymbol{h}}(n)=$ $\boldsymbol{h}(n)+\boldsymbol{v}(n)$ on a slot by slot basis ( $n$ is here the slot temporal index), where $\boldsymbol{h}$ and $\boldsymbol{v}$ are mutually uncorrelated, their components are uncorrelated and the variance of the components of $v$ depends on the training symbol power, and on the SINR.

These estimates are then refined by Wiener filtering across slots that performs the optimal compromise between temporal decorrelation due to Doppler spread and slot-wise estimation error. The refined estimate $\hat{\boldsymbol{h}}(n)$ is of the form $\hat{\boldsymbol{h}}(n)=H(q) \hat{\boldsymbol{h}}(n)$ where $H(q)$ represents the optimal Wiener filter (of unlimited order). Each component of the channel estimate becomes $\left(\hat{h}_{k, n}\right.$, component $k$, slot $\left.n\right)$

$$
\begin{aligned}
\hat{h}_{k, n} & =H_{k}(q) \hat{h}_{k, n} \\
H_{k}(q) & =\frac{1}{\left(S_{h_{k} h_{k}}(q)+\sigma_{v_{k}}^{2}\right)^{+}}\left\{\frac{S_{h_{k} h_{k}}(q)}{\left(S_{h_{k} h_{k}}(q)+\sigma_{v_{k}}^{2}\right)^{-}}\right\}_{+}
\end{aligned}
$$

where $S_{x x}(q)$ is the power spectral density function (psdf) of $x,\{\bullet\}_{+}$means "take the caisal part of" and $S_{x x}(q)=$ $S_{x x}^{+}(q) S_{x x}^{-}(q)$ is the spectral factorisation of $S_{x x}(q)$ in its causal minimum-phase factor and in its anti-causal maximum-phase counterpart. It turns out that, for an $\mathrm{AR}(1)$ model for the channel amplitudes given by $\boldsymbol{h}(n)=$ $\frac{\sqrt{1-\rho^{2}}}{1-\rho q^{-1}} \Delta \boldsymbol{h}(n)$ (where $q^{-1}$ denotes the delay operator and $\rho$ the AR forgetting factor), the psdf of $\hat{h}_{k}(n)$ is

$$
\begin{aligned}
S_{\hat{h}_{k} \hat{h}_{k}}(q) & =S_{h_{k} h_{k}}(q)+\sigma_{v_{k}}^{2} \\
& =\sigma_{v_{k}}^{2} \frac{a_{k}\left(1-b_{k} q^{-1}\right)\left(1-b_{k} q\right)}{\left(1-\rho q^{-1}\right)(1-\rho q)}
\end{aligned}
$$

where

$$
\begin{array}{ll}
b_{k} & =\left(\bar{J}_{k}-\sqrt{\left(\bar{J}_{k}\right)^{2}-4 \rho^{2}}\right)(2 \rho)^{-1} \\
a_{k}=\frac{\rho}{b_{k}} & =\frac{2 \rho^{2}}{\bar{J}_{k}-\sqrt{\left(\bar{J}_{k}\right)^{2}-4 \rho^{2}}} \\
\bar{J}_{k} & =1+\rho^{2}+\left(1-\rho^{2}\right) J_{k} \\
J_{k} & =\frac{\sigma_{h_{k}}^{2}}{\sigma_{v_{k}}^{2}}
\end{array}
$$


Hence

$$
\begin{aligned}
S_{\hat{h}_{k} \hat{h}_{k}}^{+}(q) & =\sigma_{v_{k}} \sqrt{a_{k}} \frac{1-b_{k} q^{-1}}{1-\rho q^{-1}} \\
\left\{\frac{S_{h_{k} h_{k}}(q)}{S_{\hat{h}_{k} \hat{h}_{k}}^{-}}\right\}_{+} & =\left\{\frac{\gamma_{k} q}{1-b_{k} q}+\frac{\beta_{k}}{1-\rho q^{-1}}\right\}_{+} \\
& =\frac{\beta_{k}}{1-\rho q^{-1}}
\end{aligned}
$$

where

$$
\beta_{k}=\frac{\left(1-\rho^{2}\right) \sigma_{h_{k}}^{2}}{\sigma_{v_{k}} \sqrt{a_{k}}\left(1-b_{k} \rho\right)} \quad \gamma_{k}=\beta_{k} b_{k}
$$

So we have

$$
\begin{aligned}
H_{k}(q) & =\frac{\beta_{k}}{\sigma_{v_{k}} \sqrt{a_{k}}\left(1-b_{k} q^{-1}\right)} \\
\Rightarrow \hat{\hat{h}}_{k, n} & =b_{k} \hat{\hat{h}}_{k, n-1}+\frac{\beta_{k}}{\sigma_{v_{k}} \sqrt{a_{k}}} \hat{h}_{k, n}
\end{aligned}
$$

Finally

$$
\frac{\beta_{k}}{\sigma_{v_{k}} \sqrt{a_{k}}}=\frac{\sigma_{h_{k}}^{2}}{\sigma_{v_{k}}^{2}} \frac{\left(1-\rho^{2}\right)}{\rho\left(1-b_{k} \rho\right)} b_{k}=\alpha_{k}
$$

therefore

$$
\hat{\hat{h}}_{k, n}=b_{k} \hat{\hat{h}}_{k, n-1}+\alpha_{k} \hat{h}_{k, n}
$$

where $b_{k}$ is given in (7). As a special case, when there is no time correlation over slots $(\rho=0)$, we can see that $b_{k}=0$ and $\alpha_{k}=\frac{\sigma_{h_{k}}^{2}}{\sigma_{h_{k}}^{2}+\sigma_{v_{k}}^{2}}$, so every channel component is weighted by a coefficient smaller then 1, weighting between a priori variance information and estimation error. We furthermore proposed adaptive filtering techniques (RLS) to implement the optimal filtering. So the refined estimate component $k, \hat{h}_{k, n}$, is of the form $\hat{h}_{k, n}=$ $\hat{b}_{k} \hat{h}_{k, n-1}+\hat{\alpha}_{k} \hat{h}_{k, n}$ where the two coefficients $\hat{b}_{k}$ and $\hat{\alpha}_{k}$ are given by the recursive solution

$$
\left\{\begin{array}{l}
\boldsymbol{R}_{n}=\lambda \boldsymbol{R}_{n-1}+\operatorname{Re}\left\{\left[\begin{array}{c}
\hat{\hat{h}}_{k, n-1} \\
\hat{h}_{k, n}
\end{array}\right]\left[\begin{array}{c}
\hat{\hat{h}}_{k, n-1} \\
\hat{h}_{k, n}
\end{array}\right]^{H}\right\} \\
\boldsymbol{P}_{n}=\lambda \boldsymbol{P}_{n-1}-\left[\begin{array}{c}
0 \\
\sigma_{v_{k}}^{2}
\end{array}\right] \\
\boldsymbol{\theta}_{n}=\left[\begin{array}{c}
\hat{b}_{k} \\
\hat{\alpha}_{k}-1
\end{array}\right]=\boldsymbol{R}_{n}^{-1} \boldsymbol{P}_{n}
\end{array}\right.
$$

where $\operatorname{Re}\{\}$ means "take real part of". Since the number of coupled parameters is only two, one may as well invert the $2 \times 2$ matrix rather than using true RLS. Initialisation of the algorithm requires only $R_{0}$ to be different from zero, so we can set it to $R_{0}=10^{-3} \boldsymbol{I}$; furthermore, $\hat{\boldsymbol{h}}_{k, 0}=\hat{\boldsymbol{h}}_{k, 1}$.

For structured multipath channels, we introduce 2D filtering in which the refined FIR channel estimates are approximated by a multipath model in every slot via the Recursive Early-Late algorithm, see [8].This technique derives from the basic Early-Late approach, and corresponds here to applying the Matching Pursuit technique to the convolution of the refined FIR estimate of the overall channel $\hat{h}_{k, n}$ and the pulse-shape matched filter $\left(p_{-k}^{*}=p_{k}\right.$ for the Root Raised Cosine). REL corresponds to maximum likelihood if the noise is Gaussian and white and no other users are present [1]. The sampling rate discrete-time channel impulse response during transmission of slot $n$ can be written as $h_{k, n}=\sum_{i=1}^{L} g_{i, n} p_{k-\tau_{i, n}}$. When reoptimization is done only for the amplitude of the current iteration, we can formulate the algorithm as following (define $f_{k}^{0}=f_{k}=\hat{h}_{k, n} * p_{-k}^{*}$ and $\left.q_{k}=p_{k} * p_{-k}^{*}\right)$ :

$$
\begin{aligned}
& \text { REL for } i=1, \cdots, L \quad \text { or untill } \frac{\left\|f_{n}^{i}\right\|^{2}}{\left\|f_{n}\right\|^{2}}<\beta \\
& \qquad \begin{aligned}
\tau_{i, n} & =\arg \max _{k}\left|f_{k}^{i-1}\right|^{2} \\
g_{i, n} & =f_{\tau_{i, n}} / q_{0} \\
f_{k}^{i} & =f_{k}-\sum_{l=1}^{i} g_{l, n} q_{k-\tau_{l, n}}=f_{k}^{i-1}-g_{i, n} q_{k-\tau_{i, n}}
\end{aligned} \\
& \text { end }
\end{aligned}
$$

where, for example, $\beta=0.1$

\section{1: Covariance Matrices Estimation}

With the estimated $g_{i, n}$ and $\tau_{i, n}$ we finally obtain the estimated channel $h_{k, n}^{e s t}=\sum_{i=1}^{L} g_{i, n} p_{k-\tau_{i, n}}$ and we can build the estimated received signal covariance matrix $\widehat{R}_{Y Y, n}=$ $\widehat{\sigma}_{v, n}^{2} I+\widehat{\sigma}_{t o t, n}^{2} \mathcal{T}\left(\boldsymbol{h}_{n}^{e s t}\right) \mathcal{T}^{H}\left(\boldsymbol{h}_{n}^{e s t}\right)$ needed for max SINR equalizers construction. $\widehat{\sigma}_{v, n}^{2}, \widehat{\sigma}_{t o t, n}^{2}$ can be obtained by covariance matching between the $\widehat{R}_{Y Y}$ above and a sample covariance estimate. The covariance matching can be limited to a small fraction of $R_{Y Y}$ (since we expect this estimation to work well, true $\sigma_{v}^{2}, \sigma_{t o t}^{2}$ are used in simulations). The noise model $R_{V V}=\sigma_{v}^{2} I$ can be extended to a more elaborate parsimonious model (e.g. banded block Toeplitz) [3]. The slot-wise periodicity can be replaced by another period.

\section{7: Simulations}

To evaluate the SINR loss due to channel estimation with respect to its theoretical expression (perfect channel knowledge) for each receiver structure and the channel estimation error (NMSE), we performed various simulations, with different set of parameters. All the $K$ users are considered synchronous and use the same spreading factor SF. The UMTS chip rate is assumed (3.84 Mchips/sec) and an oversampling factor of $M=2$ is used in the simulations. Here we present results for 2 different vehicular scenarios, both with 4 propagation paths of exponentially-decaying intensity profile at high speed $(120 \mathrm{~km} / \mathrm{h})$, but one with $20 \%$ of training symbols, while the other with $100 \%$ (training plus data). In the figures below, "REL" refers to channels estimated with the procedure in section 6, "max-SINR" referes to an unstructured FIR equalizer of section 3, and "mSINR ALT" refers to a structured equalizer with alternating optimization. 


\section{8: Conclusions}

We introduced structured equalizers for the equalizer-correlator downlink receiver, permitting interesting complexity-performance trade-offs. We introduced polynomial expansion on top of the equalizer-correlator receiver to permit further complexity-performance trade-offs. We introduced (temporal) Wiener filtering (and adaptive determination of its parameters) to permit optimal trade-off between temporal correlation exploitation and slot-wise estimation noise reduction. This permits significant channel estimation improvement. We suggested channel-based covariance estimation.

\section{References}

[1] H. Boujemâa and M. Siala. On a Maximum Likelihood Delay Acquisition Algorithm. In Proc. ICC 2001, Helsinki, Finland, June 2001.

[2] I. Ghauri and D. T. M. Slock. Linear receivers for the DSCDMA downlink exploiting orthogonality of spreading sequences. In Proc. 32nd Asilomar Conf. on Signals, Systems \& Computers, Pacific Grove, CA, November 1998.

[3] M. Jansson and B. Ottersten. Structured Covariance Matrix Estimation: A Parametric Approach. In Proceedings IEEE International Conference on Acoustics, Speech, and Signal Processing, pages 3172-3175, Istanbul, Turkey, June 2000.

[4] M. Lenardi, A. Medles, and D. T. M. Slock. A SINR Maximizing 2D RAKE Receiver for Multi-Sensor WCDMA Mobile Terminals . In Proc. VTC 2001 Spring, Rhodes, Greece, May 2001.

[5] M. Lenardi, A. Medles, and D. T. M. Slock. Intercell Interference Cancellation at a WCDMA Mobile Terminal by Exploiting Excess Codes. In Proc. VTC 2001 Spring, Rhodes, Greece, May 2001.

[6] M. Lenardi and D. T. M. Slock. A RAKE Receiver with Intracell Interference Cancellation for a DS-CDMA Synchronous Downlink with Orthogonal Codes. In Proc. VTC 2000 Spring, Tokyo, Japan, May 2000.

[7] M. Lenardi and D. T. M. Slock. SINR Maximizing Equalizer Receiver for DS-CDMA. In Proc. EUSIPCO 2000, Tampere, Finland, September 2000.

[8] M. Lenardi and D. T. M. Slock. Channel Estimation for a Discrete-Time RAKE Receiver in a WCDMA Downlink: Algorithms and Repercussions on SINR. In Proc. VTC 2001 Fall, Atlantic City, NJ, October 2001.

[9] M. Lenardi and D. T. M. Slock. Estimation of Time-Varying Wireless Channels and Application to the UMTS W-CDMA FDD Downlink. In submitted to European Wireless 2002, Florence - Italy, February 2002.

[10] S. Moshavi, E. Kanterakis, and D. Shilling. Multistage linear receivers for ds-cdma systems. Int. J. of Wireless Information Networks, 3(1), 1996.

[11] M. D. Zoltowski and J. S. Goldstein. Structured MMSE Equalization for Synchronous CDMA with Sparse Multipath Channels. In Proc. ICASSP 2001, Salt Lake City, UT, May 2001.

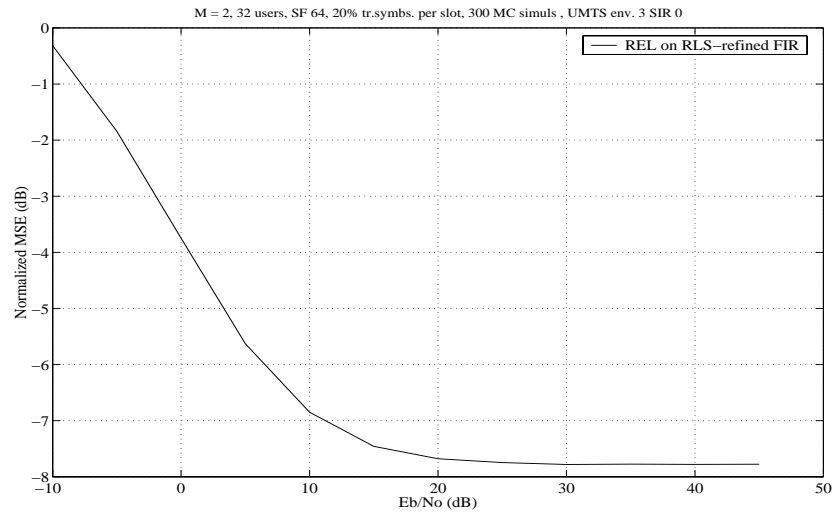

Figure 6. NMSE: vehicular, 32 users, $20 \% \operatorname{tr}$

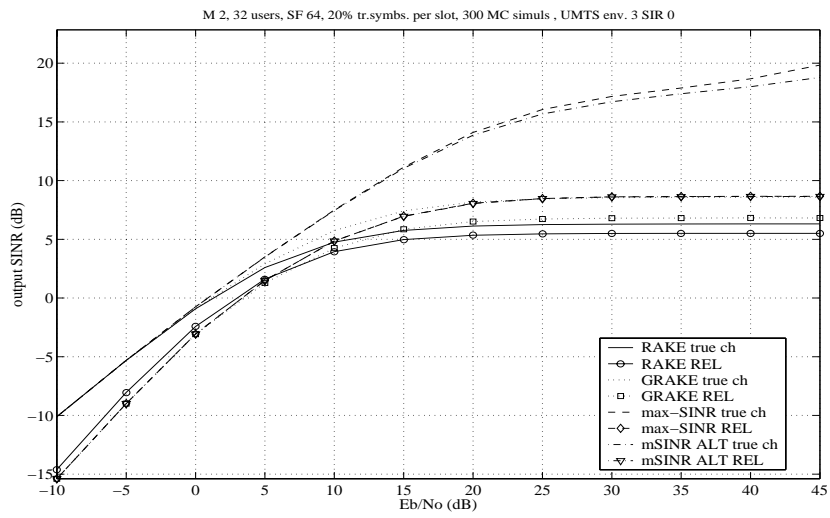

Figure 7. SINR: vehicular, 32 users, $20 \%$ tr

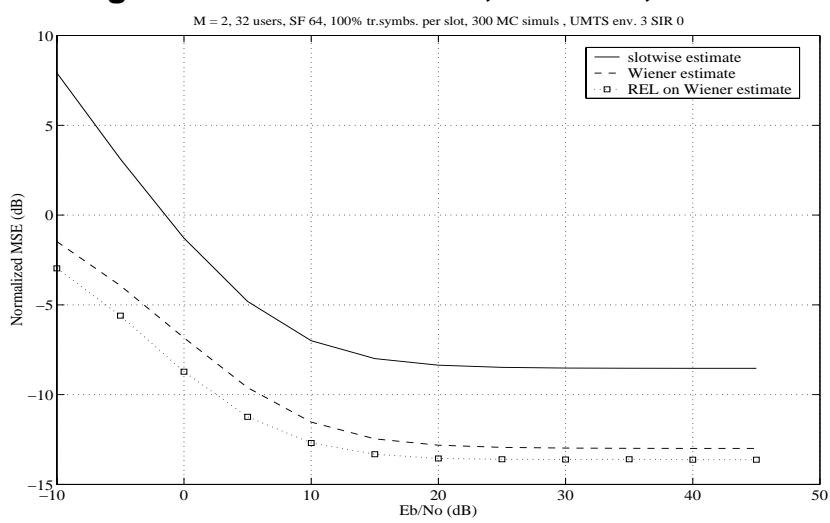

Figure 8. NMSE: vehicular, 32 users, $100 \%$ tr

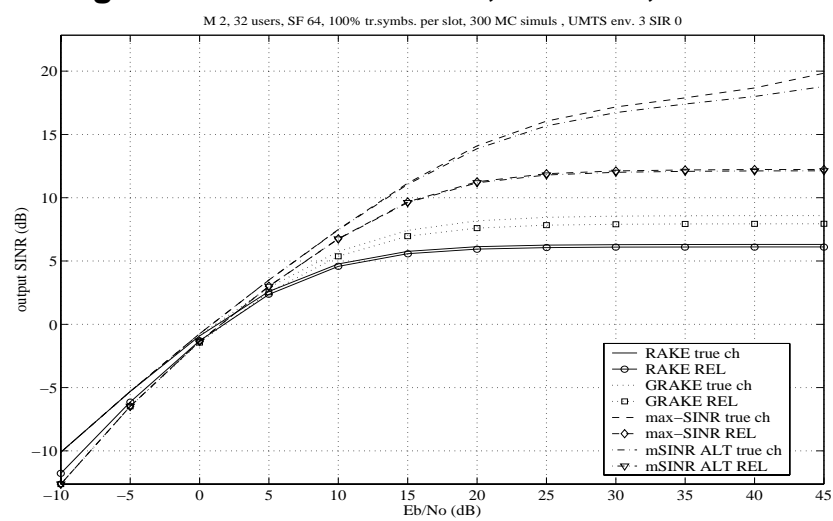

Figure 9. SINR: vehicular, 32 users, $100 \%$ tr 